\title{
Florian Vaßen (Hg.) (2010), Korrespondenzen
}

\author{
Theater. Ästhetik. Pädagogik. Berlin, Schibri Verlag.
}

\section{Gert Hofmann}

Die sogenannte „performative Wende“, der Paradigmenwechsel ästhetischer Reflexion vom Werk zum Prozess, vom künstlerischen oder literarischen Objekt zu Akt, event, happening, Aktion - kurz, zur performance - ist eine der bestimmenden Thematiken im Theoriediskurs der jüngst vergangenen Dekaden. Was das Verhältnis von Theater und Theaterpädagogik betrifft, hat dieser Paradigmenwechsel überraschenderweise erst relativ spät Raum gegriffen, obwohl ja gerade hier, im Spannungsfeld unterschiedlicher Handlungsformen, von gesellschaftlicher Praxis einerseits und künstlerischer Poiesis andererseits, die fruchtbarsten Auswirkungen zu erwarten sind.

Florian Vaßen hat nun mit dem von ihm herausgegebenen Band zum ersten Mal die sich mit diesem Paradigmenwechsel ergebenden „Korrespondenzen“ zwischen künstlerischer und pädagogischer Praxis auf dem Felde des Theaters in den Mittelpunkt der Diskussion gestellt. „Dabei geht es nicht um eine „Entästhetisierung' des Theaters, bzw. um eine ,Entpädagogisierung ' der Theaterpädagogik, sondern um ein neues Verständnis von Kunst und Pädagogik als ästhetisches Handeln und Reflektieren." (7) Was der Herausgeber hier unmissverständlich und konzis formuliert, dass es nur um die Möglichkeiten einer gleichsam ästhetischen Potenzierung pädagogischen Handelns gehen kann, nicht um eine Instrumentalisierung theatraler Kunst für die Ziele einer wie auch immer beschriebenen pädagogischen Pragmatik, liegt als Argumentationsprinzip nicht allen in diesem Band enthaltenen Artikeln zugrunde. Daher lassen sich die Beiträge durchaus einteilen in solche, die im Lichte des genannten Paradigmenwechsels kreative Interferenzen künstlerischen und pädagogischen Handelns erforschen, und solche, die aus der Perspektive eines dezidiert pädagogischen Interesses das performativ erweiterte Theaterverständnis für die Bildungpraxis erschließen wollen. Zu letzteren gehören beispielsweise die Beitäge von Ulrike Hentschel, die „Modi der Bezugnahme von Theaterpädagogik auf theatrale und gesellschaftliche Wirklichkeiten" untersucht und Dorothea Hillinger, die die Ästhetik des Bauhauses als „Modell für die Theaterpädagogik“ nutzbar machen möchte. Patrick Primavesi stellt in diesem Zusammenhang eine interessante Diskursvariante vor, indem er, u. a. mit Rekurs auf Walter Benjamins Programm eines proletarischen Kindertheaters die Umkehrung der pädagogischen Handlungsintentionalität in Tim Etchells That Night Follows Day untersucht, in welchem die Kinder sich selbst - und den anderen - die Welt der Erwachsenen zu erklären unternehmen. 
Besonders hier wird deutlich, dass die Theaterpädagogik auch in ,postdramatischen' Theaterzeiten nicht ohne eine klare Abgrenzung gegenüber der Theaterkunst auskommt, trotz aller Korrespondenzen, Interferenzen und Aneignungen. Freilich hat auch der performative Paradigmenwechsel, der die Begegnung von Pädagogik und Theaterkunst so nachhaltig inspiriert, nicht erst im 20. Jahrhundert stattgefunden. Die Schwelle zwischen künstlerischem und gesellschaftlichem, i.e. politischem oder pädagogischem Handeln, die hier überschritten wird, markiert den Eintritt in den Diskurs über das Theater in der Europäischen Kulturgeschichte überhaupt. Aristoteles hatte in seiner Poetik das Theater oder Drama unter die „poetischen“ Künste eingereiht, wobei er unter ,poetisch' genau das verstand, was wir heute als ,performativ bezeichnen, unter Einschluss z.B. der Tanzkunst, der Instrumentalmusik und des Dithyrambus. Alle diese Künste hatten bereits für Aristoteles den gemeinsamen Zug, in physisch handelnder Weise menschliches Handeln, oder Aspekte davon, nachzuahmen. Und er betonte die gesellschaftspolitische Bedeutung der „Katharsis“ als einer ebenso ekstatischen wie reflektierten Gemeinschaftserfahrung, in der das Theater kulminiert. Im vorliegenden Band sind es vor allem die Beiträge zum Genre des „Community“-Theaters, z.B. von Manfred Schewe und Gerhard Fischer, die diesem Aspekt der Theatererfahrung als gemeinschaftsinduziertem Ereignis Rechnung tragen.

Die Wahrnehmung jener Affinität von künstlerischer und gesellschaftsbezogener Praxis, welche den gegenwärtigen Theaterpädagogikdiskurs so fruchtbar macht, regt sich also bereits, von Aristoteles bezeugt, an der klassischen Wiege unserer Theatertradition. Allerdings hob Aristoteles zugleich auch den Unterschied hervor, der künstlerische (poiesis) von gesellschaftlicher Praxis kategorisch trennt. Poiesis ahmt nicht menschliche Wirklichkeit nach, wie sie ist, sondern wie sie sein könnte. Kunst zielt auf die Möglichkeiten des Allgemeinmenschlichen - ohne Rücksicht auf die faktischen Bedingungen gesellschaftlicher Realität, vielmehr als gleichsam fundamentalkritische Erfahrung ihrer Fragilität. Theaterkunst als Praxis und Erfahrung sprengt gesellschaftliche Wirklichkeit. Pädagogik als primär erfahrungs- und gesellschaftsintegrierendes Handeln (und als „Erfahrungswissenschaft“, Vaßen 7), kann sich einen derart radikalen Vorstoß ins Visionäre und Ekstatische menschlicher Wirklichkeitserfahrung nicht erlauben. Kunst kann nicht vollständig auf die notwendigerweise auch affirmativen und integrativen Ziele der Pädagogik verpflichtet werden. Es sind gerade die theoretisch avanciertesten Beiträge zu diesem Band, die auf diese Frage der Differenzierung einer jeweils unterschiedlichen Wirklichkeitserfahrung, bzw. auf die Problematik von deren Kompatibilität oder Inkompatibilität im Kontext künstlerischen und pädagogischen Handelns abheben. Hervorzuheben sind dazu vor allem die Beiträge von Florian Vaßen („Lernen und Üben“), Hajo Kurzenberger (beide aus einer eher pädagogisch akzentuierten Perspektive) und Ingrid Hentschel (eher fokusiert auf das Theater als künstlerischen Akt). Ingrid Hentschel gelingt es schließlich, im Rekurs auf die Ideen des „Deep Play“ im theatralen Ereignis einerseits die spezifische Erfahrungsintensität künstlerischer Ekstase herauszustellen, die es von jeder 
pädagogischen Absicht trennt, andererseits den radikalen Wirklichkeitsbezug erkennbar werden zu lassen, der es für pädagogisches Handeln nutzbar macht.

Florian Vaßens Band hat das Verdienst Theorie und Praxis der Theaterpädagogik im Diskurs mit theatraler Ästhetik und Kunst in eine neue Dimension gestellt zu haben. Viele wichtige Fragen werden hier eröffnet, Antworten nur zurückhaltend und nicht ohne Selbstwidersprüche gegeben - aber auch das kann ein Verdienst sein, wenn ein Diskurs im Entstehen begriffen ist. 